\title{
Quasimodular instanton partition function and the elliptic solution of Korteweg-de Vries equations
}

\author{
Wei He1 \\ Instituto de Física Teórica, Universidade Estadual Paulista, \\ Barra Funda, 01140-070, São Paulo, SP, Brazil
}

\begin{abstract}
The Gauge/Bethe correspondence relates Omega-deformed N=2 supersymmetric gauge theories to some quantum integrable models, in simple cases the integrable models can be treated as solvable quantum mechanics models. For SU(2) gauge theory with an adjoint matter, or with 4 fundamental matters, the potential of corresponding quantum model is the elliptic function. If the mass of matter takes special value then the potential is an elliptic solution of $\mathrm{KdV}$ hierarchy. We show that the deformed prepotential of gauge theory can be obtained from the average densities of conserved charges of the classical KdV solution, the UV gauge coupling dependence is assembled into the Eisenstein series. The gauge theory with adjoint mass is taken as the example.
\end{abstract}

\section{Introduction}

It is well-known that the Korteweg-de Vries equation(KdV) is an integrable Hamiltonian system of infinite dimension [1]. The rapidly decreasing solutions are obtained by the inverse scattering method [2], where the scattering data of the Schrödinger operator with the initial

\footnotetext{
${ }^{1}$ weihephys@gmail.com
} 
value $\mathrm{KdV}$ function $u(x, t=0)$ as the potential can be used to reconstruct the exact potential. In the case of reflectionless scattering, the rapidly decreasing soliton solutions are obtained.

The analogous problem of solving KdV equations with periodic initial condition leads to the discovery of relations to some other topics, including the elliptic functions, algebraic geometric methods and the finite gap spectrum problem [3, 4]. The inverse problem for the periodic potential is related to the spectral problem of Schrödinger operator with Dirichlet boundary condition. The periodic potentials are sometimes called periodic KdV soliton, albeit many aspects of the solution do not parallel with the fast decaying solitons.

The KdV system is a classical Hamiltonian system, a question we can ask is how to quantize the KdV system, and especially how to quantize the soliton solution? For the first half of the question, it was noticed that the Poisson bracket of the KdV hierarchy is the large central charge limit of the Virasoro algebra [5], therefore the conformal field theory (CFT) provides a well defined framework to deform the classical KdV system where the inverse of central charge is the deformation parameter. In the literature, the deformation procedure based on CFT is often called "quantization" because the procedure is similar to quantum mechanics deforming classical mechanics. In fact, the quantum Hamiltonians of some soliton equations, including the closely related sine-Gordon, $\mathrm{KdV}$, and $\mathrm{mKdV}$ equations, can be constructed from CFT energy momentum tensor and its derivatives, they are in involution with respect to the Virasoro algebra, and quantum soliton equations can be formally defined in this context [6, 7, 8]. These discoveries finally cumulate to a series of papers studying the integrable structure of CFT, starting from [8]. The second half of the question is also well motivated. The quantum behavior of soliton shows some remarkable properties such as particle-soliton duality, which are absent at the classical level. In $1+1$ dimension we have the example of the sine-Gordon/Thirring model duality [9], in higher dimension we have the Montonen-Olive duality for gauge theories [10].

In this paper we are interested in "quantization" of the periodic solutions mentioned above. However, there is not a first principle method to identify the corresponding quantity in CFT. We have a hint from the linear equation with periodic potential associated to $\mathrm{KdV}$ equation. This equation can be viewed from two directions. Firstly, according to the Gauge/Bethe correspondence such equation is a Schrödinger equation for some quantum mechanical models which are related to the $\Omega$-deformed 4-dimensional $\mathrm{N}=2$ supersymmetric Yang-Mills gauge theories in the Nekrasov-Shatashvili (NS) limit $\epsilon_{1} \neq 0, \epsilon_{2}=0$ [11]. On the other hand, the linear equation is a classical limit of the null operator decoupling equation of certain Liouville CFT correlation function on surfaces, see e.g. [12]. Conformal blocks of Liouville CFT are related to Nekrasov partition functions of the mentioned above $\Omega$ deformed $\mathrm{N}=2$ supersymmetric gauge theories, according to the Alday-Gaiotto-Tachikawa 
(AGT) correspondence [13]. Therefore, with these relations we can relate some periodic KdV solutions to certain CFT on particular surfaces, and also related them to some deformed $\mathrm{N}=2$ quantum gauge theory models.

We use a particular example to examine in detail the relations stated above. The periodic $\mathrm{KdV}$ solution is the Lamé potential which is a prototype of elliptic KdV soliton [3, 4], the linear equation describes quantum particle in the elliptic potential [11], the associated equation of CFT is the null vector decoupling equation for the 2-point function on the torus [13], and the gauge theory is the $N=2^{*}$ theory [14, 15, 16].

The results of the paper can be divided into two parts. In the first part, we first compute the eigenvalue function of the Schrödinger equation from classical KdV Hamiltonians of elliptic solution (10), this method is different from the commonly used WKB perturbation. Then according to the Gauge/Bethe correspondence, the spectral solution of the Schrödinger problem gives the twisted superpotential of $\epsilon_{1}$ deformed gauge theory, which is the prepotential of $\Omega$-deformed gauge theory in the NS limit. Therefore we obtain the $\epsilon_{1}$ deformed prepotential from classical KdV Hamiltonians, with all $q$ dependence contained in polynomials of Eisenstein series, in formula (17). In the second part we study the correspondence without taking the limit $\epsilon_{2} \rightarrow 0$. The computation is based on the Nekrasov partition function for generic parameter $\epsilon_{1}, \epsilon_{2}$, we propose a generalized spectral relation given in (28), then reverse the relation we get a deformed version of $\mathrm{KdV}$ charges (30). As the leading order of them are the classical charges, and they contain subleading corrections, therefore we interpret them as the quantum charges of elliptic KdV solution. Similar to the $\epsilon_{1}$ deformed case in the first part, here we can write $\epsilon_{1}, \epsilon_{2}$ deformed prepotential in a form that the coefficients are quasimodular forms, presented in (33). The quasimodular expansion of prepotential has been studied by other methods, including solving the holomorphic/modular anomaly equation, for the undeformed Seiberg-Witten theory in [17], for the deformed theory in [18, 19, 20], and performing the WKB perturbation for the elliptic potential when $\epsilon_{2}=0$ [21, 22]. Our approach gives a new perspective on its origin.

There is another elliptic potential, the Treibich-Verdier potential, which can be studied in the same way. At the moment it is not clear to us if the connections can be generalized to other KdV solutions, this is remained for future work.

The organization of the paper is as follows. In Section 2, we compute the average densities of conserved charges for the Lamé elliptic potential, from which we obtain the quasimodular form of prepotential of $N=2^{*} \mathrm{SYM}$ in the Omega background in NS limit. In Section 3 we briefly explain the connection of conformal field theory and quantization of KdV system. In Section 4, based on the AGT connection we use CFT/gauge theory technique to compute the spectrum of elliptic solution which is naturally interpreted as quantum spectrum of the 
solution. Section 5 is devoted to some open questions.

\section{Classical spectrum of elliptic solution of $\mathrm{KdV}$ equa- tions}

\subsection{From gauge theory to Schrödinger operator and KdV}

Our story starts from the Gauge/Bethe correspondence which relates the Coulomb vacuum of $\mathrm{N}=2$ supersymmetric gauge theories with $\left(\epsilon_{1}=\epsilon, \epsilon_{2}=0\right)$ deformation to the Bethe solution of some quantum integrable models [11]. In some simple cases, the $\mathrm{SU}(2)$ gauge theories are related to one particle quantum mechanical models with periodic potentials,

$$
\left(\partial^{2}-u\right) \Psi(x)=\lambda \Psi(x), \quad u(x)=u(x+T) .
$$

In these cases we have analytical results about both gauge theory and quantum mechanics models, we can precisely test the idea of the Gauge/Bethe correspondence. An important relation is about the eigenvalue $\lambda$ of Schrödinger operator and the deformed prepotential $\mathcal{F}\left(\epsilon_{1}\right)$ of gauge theory. The precise relation depends on models. The Floquet theorem indicates the monodromy of the wave function along the period $T$ takes the form $\Psi(x+T)=$ $\exp (i \nu(\lambda) T) \Psi(x)$. A key point here is that the spectrum of Schrödinger operator is given by the inverse of the function $\nu(\lambda)$, therefore we can solve the spectral problem if we can compute the function $\nu(\lambda)$.

We can follow conventional way to perform the WKB analysis for the equation (1) and obtain the function $\nu(\lambda)$ without knowing solution of wave function [23]. However, here we apply a different and simpler method to compute the function $\nu(\lambda)$. The Schrödinger operator is related to the KdV hierarchy, the coefficients of large $\lambda$ asymptotic expansion of the monodromy are KdV Hamiltonians, as we will explain momentarily.

That is not the whole story, the linear equation (11) is further related to theory of integrable quantum field theory. In our story the integrable field theory is Liouville CFT [13]. A natural next step is to study what is the story for $\mathrm{KdV}$ theory if we do not take limit $\epsilon_{2} \rightarrow 0$ for the CFT/gauge theory. Indeed, without taking classical limit for the CFT a quantum $\mathrm{KdV}(\mathrm{qKdV})$ theory was developed in a series of papers, for example [6, 7, 8]. Therefore it seems that there is a deformed version of all the relations mentioned above.

The results present in this section is a limit case of the qKdV-CFT-gauge theory connection, it is the classical limit of $q \mathrm{KdV}$, which means the large central charge limit of CFT, or the NS limit of gauge theory. In the next two sections we discuss the quantum KdV solution, with proper interpretation, where it appears that similar to the classical solution the quantum spectrum also displays quasimodular structure. 


\subsection{Densities of conserved charges of elliptic solutions}

The linear operator $L=\partial^{2}-u(x, t)$ plays a central role in the theory of KdV hierarchy. In the Lax's operator formalism of KdV hierarchy, a tower of differential operators with increasing order would give the higher order KdV equations. These operators can be obtained from the formal computation of pseudo-differential operator system of Gelfand and Dickey, the equations appear as $\partial_{t_{n}} L=\left[\left(L^{(2 n-1) / 2}\right)_{+}, L\right]$, with $n=1,2,3, \cdots$. A basic fact is that $L$ is isospectral for the $\mathrm{KdV}$ solution $u$, therefore it is enough to consider the spectrum of $L=\partial^{2}-u(x)$ with $u(x)=u(x, 0)$.

There is a nice way to see how the Hamiltonians and the monodromy are related. We start from the linear system of $L$ in (1), let $\Psi(x)=\exp \left(\int^{x} v(y) d y\right)$ and substitute it into equation (1), we get the Miura transformation,

$$
v^{\prime}+v^{2}=u+\lambda
$$

In accordance with literature, we use $\partial$ or ' to denote $\partial_{x}$. Suppose the spectral parameter $\lambda \gg 1$, perform the asymptotic expansion for $v(x)$,

$$
v=\sqrt{\lambda}+\sum_{k=1}^{\infty} \frac{v_{k}}{(\sqrt{\lambda})^{k}}
$$

then we obtain all $v_{k}$ as functionals of $u$ and its derivatives, $v_{k}=v_{k}\left(u, u^{\prime}, u^{\prime \prime}, \cdots\right)$, they can be determined recursively by the Miura transformation. For fast decaying or periodic potentials, as $v_{2 k}$ are total derivatives, the nontrivial $\mathrm{KdV}$ dynamics are driven by $v_{2 k-1}$. The KdV Hamiltonians are defined as integration of the densities $v_{2 k-1}$,

$$
H_{k}=\int d x v_{2 k-1} .
$$

The first few of them are

$$
\begin{aligned}
& H_{1}=\frac{1}{2} \int d x u, \quad H_{2}=-\frac{1}{8} \int d x\left(u^{2}-u^{\prime \prime}\right), \\
& H_{3}=\frac{1}{32} \int d x\left(2 u^{3}+u^{\prime 2}+\left(u^{\prime \prime \prime}-6 u u^{\prime}\right)^{\prime}\right), \quad \cdots,
\end{aligned}
$$

they are in involution with respect to the Poisson structure of KdV. Restore the time dependence, the KdV equations are $\partial_{t_{n}} u=\left\{H_{n}, u\right\}$. The Miura transformation is related to the bi-Hamiltonian structure of KdV hierarchy.

Now let us turn to the periodic solutions of KdV equations, the initial profile $u(x, 0)$ is given by the solution of generalized $n$th stationary equation $\left\{\sum_{k=1}^{n} c_{k} H_{k}, u\right\}=0$, where $c_{k}$ are coefficients [3]. We are interested in a class of periodic solutions given by the Weierstrass 
elliptic function, in the associated linear spectral problem they are called finite gap potentials. One periodic solution is the elliptic Lamé potential,

$$
u(x)=n(n-1) \wp\left(x ; \omega_{1}, \omega_{2}\right), \quad n \in \mathbb{Z}_{+},
$$

it solves the $(n-1)$ th stationary $\mathrm{KdV}$ equation, and $n-1$ would be the number of gaps for the real spectrum of $L$, the arithmetic genus of the associated surface. The nome for the elliptic function is $q=\exp \left(i 2 \pi \frac{\omega_{2}}{\omega_{1}}\right)$.

Recall that for the Schrödinger operator $L$ with potential $u(x)$ with period $T$, the monodromy of the wave function under $x \rightarrow x+T$ is given by $\int_{y}^{y+T} d x v(x)=i \nu T$, therefore the Floquet exponent is given by

$$
i \nu=\frac{1}{T} \int_{y}^{y+T} d x v(x) .
$$

For large $\lambda$, we can use the asymptotic expansion of $v(x)$. The integration $\varepsilon_{k}=\frac{1}{T} \int_{y}^{y+T} d x v_{2 k-1}$ is the average density of Hamiltonian $H_{k}, H_{k}=T \varepsilon_{k}$. For elliptic function there are two periods, as we have examined the large $\lambda$ asymptotic expansion is associated to $T=2 \omega_{1}$ [23]. The other period is related to dual expansion which is not suitable for the $\mathrm{KdV}$ formalism.

Substitute the Lamé potential into the Hamiltonians, we can simplify all the integrands $v_{2 k-1}(x)$ using the basic relations of the $\wp\left(x ; \omega_{1}, \omega_{2}\right)$ function and discard total derivative terms. The final form of the integrands includes two parts, one part is $x$-independent and the other part is proportional to $\wp(x)$. The expansion of Floquet exponent takes the following form

$$
i \nu=\sqrt{\lambda}+\sum_{k=1}^{\infty} \frac{\varepsilon_{k}\left(n, g_{2,3}, \frac{\zeta_{1}}{\omega_{1}}\right)}{(\sqrt{\lambda})^{2 k-1}},
$$

where $g_{2}, g_{3}$ are invariants of the $\wp(x)$ function, the integration of $\wp(x)$ zeta function is $\zeta(x)$, satisfying $\partial_{x} \zeta(x)=-\wp(x)$, and its periodic shift gives $\zeta\left(x+2 \omega_{1,2}\right)=\zeta(x)+2 \zeta_{1,2}$. The average density $\varepsilon_{k}$ can be written in terms of Eisenstein series if we use the relation

$$
\frac{\zeta_{1}}{\omega_{1}}=\frac{\pi^{2}}{3} E_{2}(q), \quad g_{2}=\frac{4 \pi^{4}}{3} E_{4}(q), \quad g_{3}=\frac{8 \pi^{6}}{27} E_{6}(q)
$$

Under the $\mathrm{SL}(2, \mathbb{Z})$ transformation $E_{4}, E_{6}$ are modular forms of weight 4 and 6 , respectively, 
while $E_{2}$ is a quasimodular form. The first few $\varepsilon_{k}$ are

$$
\begin{aligned}
\varepsilon_{1}= & -\frac{\pi^{2}}{6} n(n-1) E_{2}, \\
\varepsilon_{2}= & -\frac{\pi^{4}}{72} n^{2}(n-1)^{2} E_{4}, \\
\varepsilon_{3}= & -\frac{\pi^{6}}{2160} n^{3}(n-1)^{3}\left(9 E_{2} E_{4}-4 E_{6}\right)+\frac{\pi^{6}}{180} n^{2}(n-1)^{2}\left(E_{2} E_{4}-E_{6}\right), \\
\varepsilon_{4}= & -\frac{5 \pi^{8}}{72576} n^{4}(n-1)^{4}\left(15 E_{4}^{2}-8 E_{2} E_{6}\right)+\frac{5 \pi^{8}}{1512} n^{3}(n-1)^{3}\left(E_{4}^{2}-E_{2} E_{6}\right) \\
& -\frac{\pi^{8}}{252} n^{2}(n-1)^{2}\left(E_{4}^{2}-E_{2} E_{6}\right),
\end{aligned}
$$

Compare to the WKB computation in [23], where we essentially performed the $\epsilon$-perturbation expansion, the KdV method we employ is based on the large eigenvalue expansion. The integrand from the $\mathrm{KdV}$ densities are polynomials of $u(x)$ and its derivatives, the computation is much simpler.

Now, we can use the Bethe/Gauge correspondence to derive the deformed prepotential of gauge theory from the spectral data of Schrödinger equation [11]. In the next subsection we will show that from the spectrum of classical elliptic solution we exactly recover the deformed instanton action of $\mathrm{SU}(2) N=2^{*}$ gauge theory.

\subsection{Instanton partition function in the NS limit}

We already obtained the asymptotic relation $\nu(\lambda)$, the reverse relation gives us the asymptotic spectrum of the Lamé potential. Then according the proposal in [11], $\mathrm{N}=2$ gauge theories in the limit $\epsilon_{1}=\epsilon, \epsilon_{2}=0$ are related to quantum integrable models. In our story, Schrödinger equation with the Lamé potential is related to the gauge theory is the mass deformed $\mathrm{SU}(2) \mathrm{N}=4$ super-Yang-Mills theory, i.e. $N=2^{*}$ theory. The gauge theory mass appears in the potential as $m\left(m-\epsilon_{1}\right) \wp(x)$. Because we relate the gauge theory to the $\mathrm{KdV}$ solution we demand the mass of adjoint matter takes special value so the potential satisfies a stationary KdV equation,

$$
\frac{m}{\epsilon_{1}}=n \in \mathbb{Z}
$$

As checked in detail in [23], the spectral data $\lambda, \nu$ are related to gauge theory quantities, the moduli $\tilde{\mathfrak{u}}$ and the v.e.v. of scalar field $a$, by relation 2

$$
\lambda=-\frac{8 \pi^{2} \tilde{\mathfrak{u}}}{\epsilon_{1}^{2}}, \quad \nu=\frac{2 \pi a}{\epsilon_{1}},
$$

\footnotetext{
${ }^{2}$ Compare to [23] here we have recovered the $\pi$ factors.
} 
and $\tilde{\mathfrak{u}}=\tilde{\mathfrak{u}}\left(a, m, q, \epsilon_{1}\right)$ is related to the deformed prepotential of gauge theory in the NS limit $\mathcal{F}\left(a, m, q, \epsilon_{1}\right)$ by

$$
\tilde{\mathfrak{u}}=\frac{1}{2} q \frac{\partial}{\partial q} \mathcal{F}\left(\epsilon_{1}\right)+\frac{m\left(m-\epsilon_{1}\right)}{24}\left(1-2 E_{2}\right) .
$$

Here the same $q$ is the complex UV coupling of gauge theory, the instanton expansion parameter. The second term on the right hand side of (13) is necessary to march the eigenvalue $\lambda$ and the gauge theory prepotential $\mathcal{F}\left(\epsilon_{1}\right)$. We can derive the Schrödinger equation by examining $\epsilon_{2} \rightarrow 0$ limit of the null vector decoupling equation of Liouville CFT, treating chiral half of the correlation function as the wave function, the relation (13) can be derived [22].

In order to relate the spectrum of $\mathrm{KdV}$ solution to the instanton partition function, we need to reverse the relation (8) , and get the large $\nu$-expansion for the spectral parameter,

$$
\lambda=-\nu^{2}+\sum_{k=0}^{\infty} \frac{\lambda_{k}^{c}}{\nu^{2 k}}
$$

where $\lambda_{0}^{c}=-2 \varepsilon_{1}, \lambda_{1}^{c}=\varepsilon_{1}^{2}+2 \varepsilon_{2}, \lambda_{2}^{c}=-2\left(\varepsilon_{1}^{3}+3 \varepsilon_{1} \varepsilon_{2}+\varepsilon_{3}\right), \cdots$. Substitute $\varepsilon_{k}$, we obtain

$$
\begin{aligned}
\lambda_{0}^{c}= & \frac{\pi^{2}}{3} n(n-1) E_{2}, \\
\lambda_{1}^{c}= & \frac{\pi^{4}}{36} n^{2}(n-1)^{2}\left(E_{2}^{2}-E_{4}\right), \\
\lambda_{2}^{c}= & \frac{\pi^{6}}{540} n^{3}(n-1)^{3}\left(5 E_{2}^{3}-3 E_{2} E_{4}-2 E_{6}\right)-\frac{\pi^{6}}{90} n^{2}(n-1)^{2}\left(E_{2} E_{4}-E_{6}\right), \\
\lambda_{3}^{c}= & \frac{\pi^{8}}{9072} n^{4}(n-1)^{4}\left(35 E_{2}^{4}-7 E_{2}^{2} E_{4}-10 E_{4}^{2}-18 E_{2} E_{6}\right)-\frac{\pi^{8}}{756} n^{3}(n-1)^{3}\left(7 E_{2}^{2} E_{4}-5 E_{4}^{2}-2 E_{2} E_{6}\right) \\
& +\frac{\pi^{8}}{126} n^{2}(n-1)^{2}\left(E_{2} E_{6}-E_{4}^{2}\right),
\end{aligned}
$$

This is the asymptotic spectrum of the linear Schrödinger operator $L$ [23], but now wrote in a different form: the $\epsilon_{1}$ is hidden in $n$, the $q$ is assemble into the quasimodular functions $E_{2 k}$.

Using the differential relation of the (quasi)modular functions,

$$
\begin{aligned}
& q \frac{\partial}{\partial q} \ln \eta(q)=\frac{1}{24} E_{2}, \quad q \frac{\partial}{\partial q} E_{2}=\frac{1}{12}\left(E_{2}^{2}-E_{4}\right), \\
& q \frac{\partial}{\partial q} E_{4}=\frac{1}{3}\left(E_{2} E_{4}-E_{6}\right), \quad q \frac{\partial}{\partial q} E_{6}=\frac{1}{2}\left(E_{2} E_{6}-E_{4}^{2}\right),
\end{aligned}
$$

where $\eta(q)$ is the Dedekind eta function, we can integrate (13) to recover the gauge theory 
prepotential up to a $q$-independent integration constant,

$$
\begin{aligned}
\widetilde{\mathcal{F}}\left(\epsilon_{1}\right)= & \left(a^{2}-\frac{m\left(m-\epsilon_{1}\right)}{12}\right) \ln q+2 m\left(m-\epsilon_{1}\right) \ln \eta(q)-\frac{m^{2}\left(m-\epsilon_{1}\right)^{2}}{48 a^{2}} E_{2} \\
& -\frac{1}{5760 a^{4}}\left[m^{3}\left(m-\epsilon_{1}\right)^{3}\left(5 E_{2}^{2}+E_{4}\right)-\epsilon_{1}^{2} m^{2}\left(m-\epsilon_{1}\right)^{2} E_{4}\right]+\mathcal{O}\left(a^{-5}\right)
\end{aligned}
$$

Note that $\ln \eta(q)=\frac{1}{24} \ln q-q+\mathcal{O}\left(q^{2}\right)$, therefore terms of order $m\left(m-\epsilon_{1}\right) \ln q$ cancel out.

There are two subtle points about the above formula (17) concerning $q$-independent terms. Firstly, the integration of the last three relations in (16) about modular forms introduces some $q$-independent constant terms of definitive value. This constant part is just the $q=0$ value of terms involving $E_{2 k}$ in (17), it should not be contained in the instanton part of deformed prepotential. However, we do not need to subtract it from the prepotential (17) because it precisely gives most terms of the 1-loop perturbative part of the deformed prepotential. Secondly, there is a remaining 1-loop perturbative $q$-independent piece of order $m^{2} \ln 2 a$ which cannot be determined by our integration procedure. Together with $\widetilde{\mathcal{F}}\left(\epsilon_{1}\right)$, they give the full deformed prepotential in the NS limit,

$$
\mathcal{F}\left(\epsilon_{1}\right)=\widetilde{\mathcal{F}}\left(\epsilon_{1}\right)+m\left(m-\epsilon_{1}\right) \ln 2 a .
$$

Therefore we recover almost the full prepotential in the NS limit by integrating the classical elliptic KdV solution. In the Section 4 we will discuss these points in more detail when both deformation parameters $\epsilon_{1}, \epsilon_{2}$ are turned on where the 1-loop deformed prepotential is computed by the double gamma function.

In the limit $\epsilon_{1} \rightarrow 0$, in the gauge theory we get the solution of undeformed prepotential, see the results in [17] computed from the Seiberg-Witten curve. In quantum mechanics this limit is the leading order WKB approximation, see e.g. [22]. In KdV theory this limit is the dispersionless limit, under which only one term in $v_{2 k-1}$ survives and the Hamiltonians are very simple, $H_{k} \propto \int d x u^{k}$.

\section{Quantum KdV hierarchy and CFT}

Now we keep both deformation parameters $\epsilon_{1}, \epsilon_{2}$, and look at how the $\epsilon_{2}$ deforms all the classical relations discussed previously. The full $\epsilon_{1}, \epsilon_{2}$ deformation of gauge theory [16], and its relation to CFT are already understood [13], we would study how the corresponding elliptic solution of KdV hierarchy is deformed. As we explain below, for elliptic KdV solution it is difficult to follow the already known examples to construct quantum solution which produces the elliptic solution in the classical limit.

Let us briefly recall how the KdV theory is related to CFT. We have no a prior quantization scheme in KdV theory because it is a nonlinear system without adjustable couplings. 
The clue is the symmetry algebra, there are two Poisson structures for the KdV system, one of the Poisson brackets, given by the Magri-Virasoro bracket, is just the large central charge limit of Virasoro commutation relation [5]. Therefore we can map the potential $u(x)$ to the energy-momentum operator $T(z)$ in CFT, and continue to construct operators that are commutative with $T(z)$, they are operator analogues of the Hamiltonian $H_{k}$. Consider the CFT in the complex plane, or conformal equivalently on the infinite cylinder, the quantum actions are defined through the normal ordered product of CFT energy-momentum operator and its derivatives [6, [7, 8],

$$
\begin{aligned}
& I_{1}=\int d z T(z), \quad I_{2}=\int d z: T^{2}(z):, \\
& I_{3}=\int d z: T^{3}(z):+\frac{c+2}{12}:\left(T^{\prime}(z)\right)^{2}:, \quad \ldots
\end{aligned}
$$

they commutate with respect to the Virasoro algebra, and the quantum KdV equations can be formally defined. After explicitly carrying out integration the quantum Hamiltonians $I_{n}$ are polynomials of Virasoro generators. In the classical limit, $c \rightarrow \infty$,

$$
T(z) \rightarrow \frac{c}{6} u(z), \quad[*, *] \rightarrow \frac{6 i \pi}{c}\{*, *\}
$$

we recover classical KdV theory.

Many ingredients of the classical theory have a corresponding quantum version. In classical theory the Miura transformation (2) maps two Poisson structures satisfied by $u(x)$ and $v(x)$ respectively to each other, in the quantum version it is the Feigin-Fuchs free field realization of CFT. The ordinary differential equation of the classical theory is replaced by the null vector decoupling condition of CFT which is a partial differential equation.

This construction clarifies the connection of classical and quantum symmetry algebra, but it has not yet addressed some other points of KdV theory. The soliton solution of classical $\mathrm{KdV}$ equations is an important aspect, study the quantum version of $\mathrm{KdV}$ solitons may provide further insight about them. Generally, a CFT state in the Verma module, measured by its conformal weight, would be a solution to the quantum KdV system. In order to survive in the large $c$ limit, the conformal weight should be of order $\mathcal{O}(c)$. In the full quantum theory, the classical profile may be dressed up with light excitations created by $L_{-n}$ with conformal weight of order $\mathcal{O}(1)$. At the moment it is not clear in general what kind of CFT states, in the classical limit, would give various classical solutions of KdV equations.

The CFT dynamics on the cylinder can be directly mapped to the classical KdV dynamics on the circle, as in (19), (20). A novel feature of 2-dimensional CFT is that they can be defined on Riemann surface of nontrivial topology, i.e. surface of higher genus with punctures and boundaries. What is the corresponding story for KdV theory when the CFT is on surfaces 
of nontrivial topology? There is no obvious general answer for this question. In the case of complex plane the eigenstates for the quantum Hamiltonians can be defined by the radial quantization, then eigenvalues can be computed [8]. However, in the case of nontrivial surfaces or complex plane with branch cuts, it is unclear how to define eigenstates in CFT.

Indeed, some elliptic solutions are related to CFT on nontrivial surfaces. The classical limit of null vector decoupling equation on the torus gives the Schrödinger equation with the Lamé potential, the null vector decoupling equation on the sphere leads to the Schrödinger equation with the Treibich-Verdier potential, see e.g. [12, 24, 22]. The wave function is the classical limit of the chiral half of the correlator with a degenerate operator insertion. This fact gives us some hint about the deformed story. We computed classical KdV charges from the monodromy of wave function, we can also try to compute the deformed charges from the monodromy of conformal block when moving the degenerate operator along the conjugate cycles on the surface, which corresponds to the expectation value of the Wilson loop in gauge theory [25, 26]. For example, for the torus conformal block the $\alpha$-cycle monodromy of the degenerate operator is a simple phase $\exp \left(i \frac{2 \pi a}{\epsilon_{1}}\right)$, in the classical limit it is the same as the monodromy of Schrödinger wave function (7) whose expansion (8) gives the classical KdV charges.

We should express the deformed quantities as classical part plus large $c$ corrections. The CFT, or equivalently the $\mathrm{N}=2$ gauge theory as [13] indicates, provides effective tools to compute the large central charge deformation, therefore we can interpret the subleading terms as quantum effects for the periodic solutions. The $\Omega$ background parameters of gauge theory are related to the central charge of CFT by

$$
c=1+6 \frac{\left(\epsilon_{1}+\epsilon_{2}\right)^{2}}{\epsilon_{1} \epsilon_{2}} .
$$

Therefore the NS limit $\epsilon_{1}=\epsilon, \epsilon_{2} \rightarrow 0$ in gauge theory is the classical limit of CFT, $c \rightarrow \infty$. In the case of torus 1-point block, the external state has a conformal weight

$$
\Delta_{m}=\frac{m\left(\epsilon_{1}+\epsilon_{2}-m\right)}{\epsilon_{1} \epsilon_{2}} .
$$

In the classical limit $\Delta_{m} \sim \mathcal{O}(n(n-1) c)$, hence it produces the Lamé potential. Instead of performing large $c$ expansion, we define a deformation parameter by

$$
\hbar=\frac{\epsilon_{2}}{\epsilon_{1}} .
$$

It can be views as the quantum parameter for the nonlinear KdV system because we assume $\hbar$ is small. $\epsilon_{1}$ is the quantum parameter for the associated linear equation (1), here it is used to make the coefficient of elliptic potential to be triangular numbers $n(n-1)$ in order to satisfy the stationary KdV equations. 


\section{Quasimodular instanton partition function}

The method adopt in this section gives us two interesting results. On the one hand the $\mathrm{CFT}$ /gauge theory computation gives us explicit answer how the classical KdV solution is deformed by $\epsilon_{2}$, based on the underlying Virasoro algebra. On the other hand, the prepotential $\mathcal{F}\left(\epsilon_{1}, \epsilon_{2}\right)$ of $\mathrm{N}=2$ theories from localization computation is explicitly expanded as a $q$-series, quasimodular form of the $\mathrm{KdV}$ charges helps us to rewrite the expansion as large $a$-expansion with quasimodular coefficients.

\subsection{Deformed Hamiltonians of elliptic solution}

In the deformed case we do not have explicit solution to the deformed KdV equation. Therefore, in contrary to the Section 2, now we suppose the associated CFT conformal block defines a deformation for the classical solution. However, we do not work in the framework of CFT, instead we compute them from the instanton partition function of deformed $\mathrm{N}=2$ gauge theory. Their equivalence is guaranteed by the widely confirmed AGT correspondence. We propose an explicit relation between quantum Hamiltonians of elliptic solution and quasimodular expansion of the gauge theory prepotential.

Notice that in gauge theory the $\epsilon_{2}$ deformation only modestly deforms $\mathcal{F}\left(\epsilon_{1}\right)$ as $\epsilon_{1}, \epsilon_{2}$ are symmetric deformation, therefore we go through a procedure imitates the classical case although there is not a spectral problem like (1) for the deformed KdV theory. When we relate classical $\mathrm{KdV}$ hierarchy to gauge theory we used the fact that the monodromy relation of $\mathrm{KdV}$ in equation (8) is identified with the gauge theory relation $a=a\left(\tilde{\mathfrak{u}}, m, q, \epsilon_{1}\right)$. In the deformed case, gauge theory generalizes the monodromy relation to $a=a\left(\tilde{\mathfrak{u}}, m, q, \epsilon_{1}, \epsilon_{2}\right)$, which gives the quantum monodromy of $\mathrm{KdV}$ solution. In order to perform $\hbar$ expansion, we only need to slightly change some identifications in Section 2 in order to have a smooth limit to the classical theory. The same identification is made for the following parameters,

$$
n=\frac{m}{\epsilon_{1}}, \quad \nu=\frac{2 \pi a}{\epsilon_{1}}, \quad \lambda=-\frac{8 \pi^{2} \tilde{\mathfrak{u}}}{\epsilon_{1}^{2}} .
$$

We could make $\epsilon_{1}, \epsilon_{2}$ appear symmetrically by substituting $\epsilon_{1} \rightarrow \sqrt{\epsilon_{1} \epsilon_{2}}$. But this is only a rescaling, it is not essential. We also propose a relation that naturally generalizes (13),

$$
\tilde{\mathfrak{u}}=\frac{1}{2} q \frac{\partial}{\partial q} \mathcal{F}\left(\epsilon_{1}, \epsilon_{2}\right)+\frac{\left(m-\epsilon_{1}\right)\left(m-\epsilon_{2}\right)}{24}\left(1-2 E_{2}\right) .
$$

Recall that in the case of classical KdV solution we could fix the relation (13) because we can independently compute the KdV charges. In the deformed case we can only conjecture a relation. The form of the deformed function $\mathcal{F}\left(\epsilon_{1}, \epsilon_{2}\right)$, computed from the Nekrasov partition 
function, gives us a hint about the second term on the right hand side of (25),

$$
q \frac{\partial}{\partial q} \mathcal{F}\left(\epsilon_{1}, \epsilon_{2}\right)=a^{2}-\frac{\left(m-\epsilon_{1}\right)\left(m-\epsilon_{2}\right)}{12}\left(1-E_{2}\right)+\mathcal{O}\left(a^{-2}\right)
$$

In the expansion coefficients of $a^{-2 k}$ are $\lambda_{k}^{q}, k \geqslant 1$, their quasimodular polynomials have a rigidity property, as explained below in (28). Our conjectural relation maintains this property for $\lambda_{0}^{q}$.

As in the classical limit $\epsilon_{2} \rightarrow 0$, the deformed prepotential $\mathcal{F}\left(\epsilon_{1}, \epsilon_{2}\right)$ should reduces to the deformed prepotential $\mathcal{F}\left(\epsilon_{1}\right)$ in formula (17), it is reasonable to suspect the quasimodular polynomials are preserved in someway in the $\epsilon_{2}$ deformed case. Indeed, from the Nekrasov partition function we find that for $\lambda_{2 k}^{q}$ in the series

$$
\lambda=-\nu^{2}+\sum_{k=0}^{\infty} \frac{\lambda_{k}^{q}(\hbar)}{\nu^{2 k}}
$$

we can assemble all $q$ into $E_{2 k}$, then the polynomials of Eisenstein series appearing in the classical case are preserved, only the coefficients $[n(n-1)]^{k}$ are corrected by $\hbar$. If we define $\mathbf{N}=(n-\hbar)(n-1)$, then

$$
\begin{aligned}
\lambda_{0}^{q}= & \frac{\pi^{2}}{3} \mathbf{N} E_{2}, \\
\lambda_{1}^{q}= & \frac{\pi^{4}}{36} \mathbf{N}(\mathbf{N}-\hbar)\left(E_{2}^{2}-E_{4}\right), \\
\lambda_{2}^{q}= & \frac{\pi^{6}}{540} \mathbf{N}(\mathbf{N}-\hbar)\left(\mathbf{N}+\frac{1}{2} \hbar\right)\left(5 E_{2}^{3}-3 E_{2} E_{4}-2 E_{6}\right)-\frac{\pi^{6}}{90}(1+\hbar)^{2} \mathbf{N}(\mathbf{N}-\hbar)\left(E_{2} E_{4}-E_{6}\right), \\
\lambda_{3}^{q}= & \frac{\pi^{8}}{9072} \mathbf{N}(\mathbf{N}-\hbar)\left(\mathbf{N}^{2}+\frac{7}{5} \mathbf{N} \hbar+\frac{3}{5} \hbar^{2}\right)\left(35 E_{2}^{4}-7 E_{2}^{2} E_{4}-10 E_{4}^{2}-18 E_{2} E_{6}\right) \\
& -\frac{\pi^{8}}{756} \mathbf{N}(\mathbf{N}-\hbar)\left[\mathbf{N}\left(1+\frac{9}{5} \hbar+\hbar^{2}\right)+\hbar\left(\frac{3}{2}+\frac{59}{20} \hbar+\frac{3}{2} \hbar^{2}\right)\right]\left(7 E_{2}^{2} E_{4}-5 E_{4}^{2}-2 E_{2} E_{6}\right) \\
& +\frac{\pi^{8}}{126}(1+\hbar)^{4} \mathbf{N}(\mathbf{N}-\hbar)\left(E_{2} E_{6}-E_{4}^{2}\right),
\end{aligned}
$$

From this perspective the polynomial ring of $E_{2}, E_{4}, E_{6}$ in the expansion of $\lambda$ has certain rigidity under the second deformation $\epsilon_{2}$.

We can inverse the relation (27) to get the quantum monodromy relation that gives the quantum energy densities of the periodic solution,

$$
i \nu=\sqrt{\lambda}+\sum_{k=1}^{\infty} \frac{\varepsilon_{k}^{q}\left(n, g_{2,3}, \frac{\zeta_{1}}{\omega_{1}}, \hbar\right)}{(\sqrt{\lambda})^{2 k-1}},
$$


where

$$
\begin{aligned}
\varepsilon_{1}^{q}= & -\frac{\pi^{2}}{6}\left\{n(n-1) E_{2}-\hbar(n-1) E_{2}\right\}, \\
\varepsilon_{2}^{q}= & -\frac{\pi^{4}}{72}\left\{n^{2}(n-1)^{2} E_{4}+\hbar\left[n(n-1)\left(E_{2}^{2}+E_{4}\right)-2 n^{2}(n-1) E_{4}\right]\right. \\
& \left.+\hbar^{2}\left[n(n-1) E_{4}-(n-1) E_{2}^{2}\right]\right\},
\end{aligned}
$$

$\varepsilon_{k}$ explicitly show how the classical charges are deformed. This is an exact quantization, in every $\varepsilon_{k}$ the quantum effect grows to a finite power of $\hbar$. As we do not observe interesting pattern in the expansion, we cease to give more detail on this.

\subsection{Quasimodular prepotential of gauge theory}

Let us move a step backward to retrieve the prepotential by integrating the relation (25), we get an expansion $\widetilde{\mathcal{F}}\left(\epsilon_{1}, \epsilon_{2}\right)$ which is similar to $\widetilde{\mathcal{F}}\left(\epsilon_{1}\right)$ in (17).

$$
\begin{aligned}
\widetilde{\mathcal{F}}\left(\epsilon_{1}, \epsilon_{2}\right)= & \left(a^{2}-\frac{\left(m-\epsilon_{1}\right)\left(m-\epsilon_{2}\right)}{12}\right) \ln q+2\left(m-\epsilon_{1}\right)\left(m-\epsilon_{2}\right) \ln \eta(q) \\
& -\frac{m\left(m-\epsilon_{1}\right)\left(m-\epsilon_{2}\right)\left(m-\epsilon_{1}-\epsilon_{2}\right)}{48 a^{2}} E_{2}+\mathcal{O}\left(a^{-4}\right) .
\end{aligned}
$$

From the structure of formula (28), $\widetilde{\mathcal{F}}\left(\epsilon_{1}, \epsilon_{2}\right)$ contains the same quasimodular polynomials as in $\widetilde{\mathcal{F}}\left(\epsilon_{1}\right)$, only with mass terms further deformed by $\epsilon_{2}$. The issue about the $q$-independent integration constant part is similar to the case discussed in Section 2, they are related to the perturbative part of the prepotential. Among the perturbative contribution, most of terms of order $a^{-2 n}$ are precisely the integration constant already introduced in $E_{2 k}$, another piece of perturbative contribution undetermined contains terms with $\ln 2 a$. The full deformed prepotential is

$$
\mathcal{F}\left(\epsilon_{1}, \epsilon_{2}\right)=\widetilde{\mathcal{F}}\left(\epsilon_{1}, \epsilon_{2}\right)+\left(m-\epsilon_{1}\right)\left(m-\epsilon_{2}\right) \ln 2 a
$$

The 1-loop and instanton contribution are related in a subtle way because in the Nekrasov partition function the perturbative part is represented as the regularized universal denominator from the instanton part [16], the regularized function can be computed by the logarithm of double gamma function, see e.g. [27, 19]. See A for some details.

It is instructive to write the deformed prepotential in another form, to compare with the results derived by other methods [19, 21, 18]. We need to make a shift for the mass,

$m \rightarrow m+\frac{\epsilon_{1}+\epsilon_{2}}{2}$, then the quasimodular part of the prepotential, or the terms involving $E_{2 k}$, can be expanded as

$$
\mathcal{F}_{\text {mod }}\left(a, m+\frac{\epsilon_{1}+\epsilon_{2}}{2}, q, \epsilon_{1,2}\right)=\sum_{g, h=0}^{\infty}\left(\epsilon_{1}+\epsilon_{2}\right)^{2 g}\left(\epsilon_{1} \epsilon_{2}\right)^{h} \mathbf{F}_{g, h}\left(a, m, E_{2}, E_{4}, E_{6}\right) .
$$


The $\mathbf{F}_{0,0}$ coincides with the result obtained from the Seiberg-Witten curve [17],

$$
\mathbf{F}_{0,0}=-\frac{m^{4}}{48 a^{2}} E_{2}-\frac{m^{6}}{5760 a^{4}}\left(5 E_{2}^{2}+E_{4}\right)-\frac{m^{8}}{2903040 a^{6}}\left(175 E_{2}^{3}+84 E_{2} E_{4}+11 E_{6}\right)+\mathcal{O}\left(\frac{m^{10}}{a^{8}}\right) .
$$

We list a few other $\mathbf{F}_{g, h}$ up to order $a^{-6}$ which may be useful, they are

$$
\begin{aligned}
& \mathbf{F}_{1,0}=\frac{m^{2}}{96 a^{2}} E_{2}+\frac{m^{4}}{1536 a^{4}}\left(E_{2}^{2}+E_{4}\right)+\frac{m^{6}}{414720 a^{6}}\left(25 E_{2}^{3}+48 E_{2} E_{4}+17 E_{6}\right)+\mathcal{O}\left(\frac{m^{8}}{a^{8}}\right), \\
& \mathbf{F}_{2,0}=-\frac{1}{768 a^{2}} E_{2}-\frac{m^{2}}{30720 a^{4}}\left(5 E_{2}^{2}+9 E_{4}\right)-\frac{m^{4}}{1105920 a^{6}}\left(25 E_{2}^{3}+84 E_{2} E_{4}+101 E_{6}\right)+\mathcal{O}\left(\frac{m^{6}}{a^{8}}\right), \\
& \mathbf{F}_{3,0}=\frac{1}{368640 a^{4}}\left(5 E_{2}^{2}+13 E_{4}\right)+\frac{m^{2}}{9289728 a^{6}}\left(35 E_{2}^{3}+168 E_{2} E_{4}+355 E_{6}\right)+\mathcal{O}\left(\frac{m^{4}}{a^{8}}\right),
\end{aligned}
$$

and

$$
\begin{aligned}
& \mathbf{F}_{0,1}=-\frac{m^{2}}{48 a^{2}} E_{2}-\frac{m^{4}}{2304 a^{4}}\left(5 E_{2}^{2}+E_{4}\right)-\frac{m^{6}}{41472 a^{6}}\left(11 E_{2}^{3}+6 E_{2} E_{4}+E_{6}\right)+\mathcal{O}\left(\frac{m^{8}}{a^{8}}\right), \\
& \mathbf{F}_{1,1}=\frac{1}{192 a^{2}} E_{2}+\frac{m^{2}}{23040 a^{4}}\left(25 E_{2}^{2}+17 E_{4}\right)+\frac{m^{4}}{276480 a^{6}}\left(55 E_{2}^{3}+114 E_{2} E_{4}+41 E_{6}\right)+\mathcal{O}\left(\frac{m^{6}}{a^{8}}\right), \\
& \mathbf{F}_{2,1}=-\frac{1}{184320 a^{4}}\left(25 E_{2}^{2}+29 E_{4}\right)-\frac{m^{2}}{7741440 a^{6}}\left(385 E_{2}^{3}+1386 E_{2} E_{4}+1019 E_{6}\right)+\mathcal{O}\left(\frac{m^{4}}{a^{8}}\right),
\end{aligned}
$$

The quasimodular expansion of deformed prepotential has been studied by other methods, from other perspectives. In [19, 20] the $\left(\epsilon_{1}, \epsilon_{2}\right)$ deformed prepotential is rewritten in the quasimodular form, and a recursive relation due to the quasimodular property of $E_{2}$ is found. Result for the limit case $\epsilon_{2}=0$ is related to classical CFT block, the quasimodular expansion is obtained by a WKB computation for the Lamé potential [21, 22]. The failure of exact modular of $E_{2}$ can be remedied by introducing an antiholomorphic piece, $N=2^{*}$ gauge theory is studied in the holomorphic anomaly approach in [18]. Here we do not have an independent new computation method because the deformation of the coefficient $[n(n-1)]^{k}$ is based on the computation of Nekrasov partition function, however, we propose a precise relation to the deformation of elliptic KdV solution.

\section{Conclusion and discussion}

We present an example of relating elliptic KdV solution to 4-dimensional supersymmetric gauge theory. This can be viewed as a natural result from a chain of very interesting connections, the qKdV-CFT relation [8], the CFT/Gauge correspondence [13] and the Gauge/Bethe correspondence [11]. The Schrödinger equation (1) provides a key hint, the Virasoro symmetry is the real reason that makes these connections work. Using all the relations, we obtain 
the deformed prepotential of gauge theory with the UV coupling dependence assembled into Eisenstein series, which originate from the Weierstrass elliptic function of the KdV solution. On the other hand, we get the quantum spectrum of elliptic KdV solution.

There is another elliptic KdV solution [28],

$$
u(x)=\sum_{j=0}^{3} n_{j}\left(n_{j}-1\right) \wp\left(x+\omega_{j}\right)
$$

It is related to the sphere 4-point conformal block, and $\mathrm{SU}(2) N_{f}=4 \mathrm{SYM}$ gauge theory. We emphasis unlike the Lamé potential, in the potential (37) the modulus is not the instanton/conformal block expansion parameter [24]. We have confirmed that the spectrum of the potential is related to the quasimodular expansion of the prepotential for $\mathrm{SU}(2) N_{f}=4 \mathrm{SYM}$ in the NS limit. The full Nekrasov instanton partition function would give a deformation of the spectrum. Some formulae about integration of elliptic function, useful to compute the classical spectrum in this case, has been given in [29].

It is not clear if the two examples appear just by coincidence, or in more general case some elliptic KdV solutions are related to some CFT blocks and gauge theories. There is a large class of CFT blocks in [13], on the other hand up to now the most general elliptic solution of $\mathrm{KdV}$ is the Picard potential [30]. Superconformal Liouville theory is related to gauge theory on orbifold discussed in [31, while the supersymmetric KdV is constructed in [32]. The simplest extended conformal algebra $W_{3}$ is connected to the $\mathrm{SU}(3)$ gauge theories [33], and on the other side is connected to the quantum Boussinesq equation [34], and similarly they have supersymmetric extension. For the connection of general $W$-algebra and generalized classical nonlinear equation of KdV type, see [35.

\section{A Perturbative part of the prepotential}

The perturbative prepotential includes the classical part $\mathcal{F}^{\text {clas }}=a^{2} \ln q$ and the $q$-independent 1-loop part $\mathcal{F}^{1-\text { loop }}$. The $\mathcal{F}^{1-\text { loop }}$ of the $\epsilon_{1}, \epsilon_{2}$ deformed $\mathrm{N}=2$ gauge theory can be represented as regularized universal denominator extracted from the instanton partition function [16], the function is given by the logarithm of Barnes' double gamma function [27]. For SU(2) $N=2^{*}$ theory it is given by

$$
\mathcal{F}^{1-\text { loop }}=\gamma_{\epsilon_{1} \epsilon_{2}}\left(2 a+\epsilon_{1}\right)+\gamma_{\epsilon_{1} \epsilon_{2}}\left(2 a+\epsilon_{2}\right)-\gamma_{\epsilon_{1} \epsilon_{2}}(2 a+m)-\gamma_{\epsilon_{1} \epsilon_{2}}\left(2 a-m+\epsilon_{1}+\epsilon_{2}\right)
$$

The following integral form is useful for computing the large $x$ asymptotic expansion,

$$
\gamma_{\epsilon_{1} \epsilon_{2}}(x)=\lim _{s \rightarrow 0} \frac{d}{d s} \frac{1}{\Gamma(s)} \int_{0}^{\infty} d t t^{s-1} \frac{e^{-t x}}{\left(1-e^{-\epsilon_{1} t}\right)\left(1-e^{-\epsilon_{2} t}\right)}, \quad|\operatorname{Arg}(x)|<\pi .
$$


We can compute the asymptotic expansion in the semiclassical region $a \gg m$. The term containing logarithm is simply $\left(m-\epsilon_{1}\right)\left(m-\epsilon_{2}\right) \ln 2 a$. The remaining terms are of the form $a^{-2 n} f\left(m, \epsilon_{1,2}\right), n \geqslant 1$, if we shift the mass by $m \rightarrow m+\frac{\epsilon_{1}+\epsilon_{2}}{2}$ then it expands as

$$
\sum_{g, h=0}^{\infty}\left(\epsilon_{1}+\epsilon_{2}\right)^{2 g}\left(\epsilon_{1} \epsilon_{2}\right)^{h} \mathbf{f}_{g, h}(a, m) .
$$

We indeed find $\mathbf{f}_{g, h}=\left.\mathbf{F}_{g, h}\right|_{q=0}$.

\section{Acknowledgments}

I would like to thank Andrei Mikhailov for valuable discussion and reading the draft. I also thank the referee for detailed and very helpful comments. This work is supported by the FAPESP No. 2011/21812-8, through IFT-UNESP.

\section{References}

[1] V. E. Zakharov, L. D. Faddeev, Korteweg-de Vries equation, a completely integrable Hamiltonian system, Funct. Anal. Appl. 5 (1971) 280-287.

[2] C. S. Gardner, J. M. Greene, M. D. Kruskal, R. M. Miura, Method for solving the Korteweg-de Vries equation, Phys. Rev. Letters 19 (1967) 1095-1097.

[3] S. P. Novikov, A periodic problem for the Korteweg-de Vries equation, Funct. Anal. Appl. 8 (1974) 236-246.

[4] P. D. Lax, Periodic solutions of the KdV equation, Commun. Pure Appl. Math. 28 (1975) 141-148.

[5] J.-L. Gervais, Infinite family of polynomial functions of the Virasoro generators with vanishing Poisson brackets, Phys. Lett. B 160 (1985) 277-278.

[6] R. Sasaki, I. Yamanaka, Virasoro algebra, vertex operators, quantum Sine-Gordon and solvable quantum field theories, Adv. Stud. Pure. Math. 16 (1988) 271-296.

[7] T. Eguchi, S.-K. Yang, Deformation of conformal field theories and soliton equations, Phys. Lett. B 224 (1989) 373-378.

[8] V. V. Bazhanov, S. L. Lukyanov, A. B. Zamolodchikov, Integrable Structure of Conformal Field Theory, Quantum KdV Theory and Thermodynamic Bethe Ansatz, Comm. Math. Phys. 177 (1996) 381-398. 
[9] S. Coleman, Quantum sine-Gordon equation as the massive Thirring model, Phys. Rev. D 11 (1975) 2088-2097.

[10] C. Montonen, D. Olive, Magnetic monopoles as gauge particles?, Physics Letters B 72 (1977) 117-120.

[11] N. Nekrasov, S. Shatashvili, Quantization of integrable systems and four dimensional gauge theories, in: 16th International Congress on Mathematical Physics, 2009. arXiv:0908.4052.

[12] K. Maruyoshi, M. Taki, Deformed Prepotential, Quantum Integrable System and Liouville Field Theory, Nucl.Phys. B841 (2010) 388-425.

[13] L. F. Alday, D. Gaiotto, Y. Tachikawa, Liouville correlation functions from four dimensional gauge theories, Lett. Math. Phys. 91 (2010) 167.

[14] N. Seiberg, E. Witten, Electric-Magnetic Duality, Monopole Condensation, And Confinement In N = 2 Supersymmetric Yang-Mills Theory, Nucl. Phys. B 426 (1994) 19-52.

[15] N. Seiberg, E. Witten, Monopoles, Duality and Chiral Symmetry Breaking in N=2 Supersymmetric QCD, Nucl. Phys. B 431 (1994) 484-550.

[16] N. Nekrasov, Seiberg-Witten Prepotential From Instanton Counting, Adv. Theor. Math. Phys. 7 (2004) 831-864.

[17] J. A. Minahan, D. Nemeschansky, N. P. Warner, Instanton Expansions for Mass Deformed N=4 Super Yang-Mills Theories, Nucl. Phys. B 528 (1998) 109-132.

[18] M. x. Huang, A.-K. Kashani-Poor, A. Klemm, The Omega deformed B-model for rigid $\mathrm{N}=2$ theories, Ann. Henri Poincare 14 (2013) 425-497.

[19] M. Billo, M. Frau, L. Gallot, A. Lerda, I. Pesando, Deformed N = 2 theories, generalized recursion relations and S-duality, J. High Energy Phys. 1304 (2013) 039.

[20] M. Billo, M. Frau, L. Gallot, A. Lerda, I. Pesando, Modular anomaly equation, heat kernel and S-duality in N=2 theories, J. High Energy Phys. 1311 (2013) 123.

[21] A. K. Kashani-Poor, J. Troost, The toroidal block and the genus expansion, J. High Energy Phys. 1303 (2013) 133.

[22] M. Piatek, Classical torus conformal block, $N=2^{*}$ twisted superpotential and the accessory parameter of Lamé equation, J. High Energy Phys. 1403 (2014) 124. 
[23] W. He, Combinatorial approach to Mathieu and Lamé equations. arXiv:1108.0300.

[24] W. He, N=2 supersymmetric QCD and elliptic potentials, J. High Energy Phys. 1411 (2014) 030 .

[25] L. F. Alday, D. Gaiotto, S. Gukov, Y. Tachikawa, H. Verlinde, Loop and surface operators in $\mathrm{N}=2$ gauge theory and Liouville modular geometry, J. High Energy Phys. 1001 (2010) 113.

[26] N. Drukker, J. Gomis, T. Okuda, J. Teschner, Gauge Theory Loop Operators and Liouville Theory, J. High Energy Phys. 1002 (2010) 057.

[27] N. Nekrasov, A. Okounkov, Seiberg-Witten theory and random partitions. arXiv:hep-th/0306238.

[28] A. Treibich, New Elliptic Potentials, Acta. Appl. Math. 36 (1994) 27-48.

[29] A.-K. Kashani-Poor, J. Troost, Transformations of Spherical Blocks, J. High Energy Phys. 1310 (2013) 009.

[30] F. Gesztesy, R. Weikard, Elliptic algebraic-geometric solutions of KDV and AKNS- an analytical approach, Bull. Amer. Math. Soc. 35 (1998) 271-317.

[31] V. Belavin, B. Feigin, Super Liouville conformal blocks from N=2 SU(2) quiver gauge theories, J. High Energy Phys. 1107 (2011) 079.

[32] P. Mathieu, Supersymmetric extension of the Korteweg-de Vries equation, J. Math. Phys. 29 (1988) 2499.

[33] N. Wyllard, $A_{N-1}$ conformal Toda field theory correlation functions from conformal $\mathrm{N}=2 \mathrm{SU}(\mathrm{N})$ quiver gauge theories, J. High Energy Phys. 0911 (2009) 002.

[34] V. V. Bazhanov, A. N. Hibberd, S. M. Khoroshkin, Integrable structure of $W_{3}$ Conformal Field Theory, Quantum Boussinesq Theory and Boundary Affine Toda Theory, Nucl. Phys. B 622 (2002) 475-547.

[35] L. A. Dickey, Lectures on Classical W-Algebras, Acta. Appl. Math. 47 (1997) 243-321. 